\title{
British Association of Paediatric Otolaryngology, 9 September 2005, Edinburgh Scotland, UK
}

\section{Powered endoscopic dacrocystorhinostomy in children}

S Agrawal, J Tahery, C Inkster, C Lobo

From the Departments of Otorhinolaryngology and Ophthalmology Royal Bolton Hospital, Bolton, UK

\section{Introduction}

Dacrocystorhinostomy is a commonly performed procedure by both ophthalmologists and otolaryngologists. It was traditionally performed by an external approach, and more recently endoscopic laser dacrocystorhinostomy had gained popularity. More recent studies in adult populations suggest powered endoscopic dacrocystorhinostomy offers improved success over the laser with results equalling the external approach. There is no specific paediatric data with this technique, and we present our experience.

\section{Method}

A retrospective, non-comparative case notes review of paediatric cases of endoscopic powered dacrocystorhinostomy performed between October 2001 and December 2003. Surgery was performed under general anaesthesia using a standard technique as described by Wormald in 2002. This involves using a microdebrider, raising a ' $\mathrm{D}$ ' shaped mucosal flap and making an ' $\mathrm{I}$ ' incision in the lacrimal sac. A Crawford stent is inserted, where possible, to maintain patency for the initial post-operative period.

\section{Results}

Five children with mean age eight years (range 3-14 years) underwent surgery. This was indicated for simple epiphora in two cases and for recurrent dacrocystitis in the other three cases. Two children with dacrocystitis also had Down syndrome. A stent was inserted for a mean period of six weeks in all but one child in whom complete stenosis of the superior canaliculus precluded insertion. At one year follow up, four children were asymptomatic. One child with dacrocystitis had some residual epiphora but was much improved by surgery.

\section{Conclusion}

Based on our results to date, we believe that powered endoscopic dacrocystorhinostomy is an alternative to other established techniques used in the management of epiphora or dacrocystitis in children. Our own results in adults using this technique match those of external approach dacrocystorhinostomy, the gold standard. If this can be mirrored in children, it may become the procedure of choice.

\section{High pressure water jet injury to the pharynx requiring intubation}

M Ahmad, R Capper, K Midwinter

From the Doncaster Royal Infirmary, Doncaster, UK

\section{Introduction}

High-pressure water jets are used in several industries for cleaning and cutting and can deliver fluids at very high pressures up to 4000 bar. Injuries from high-pressure water jet devices are surgical emergencies as the high velocity generated produces injuries that resemble trauma caused by high-velocity missiles. These injuries show a unique pattern of extensive internal damage, often masked by trivial external evidence of injury. We report the case of a patient who sustained a high-pressure water jet injury to the pharynx resulting in airway obstruction.

\section{Case report}

An eight-year-old boy was brought to the emergency room with neck pain, dysphagia and dysphonia. He had suffered a high-pressure injury to his oropharynx when a jet of water was sprayed into his mouth from a car jet wash. There was no stridor and oxygen saturation was 100 per cent at the time of presentation with a respiratory rate of 16 per minute. On examination, there was diffuse swelling and tenderness over the anterior neck. He was unable to speak in complete sentences. A lateral neck X-ray showed soft tissue emphysema in the prevertebral soft tissues and retropharyngeal swelling impinging on the airway. Fibre-optic examination revealed marked swelling of the posterior pharyngeal wall, nearly touching the arytenoids. The patient was intubated under general anaesthetic and transferred to the paediatric intensive care unit where he was ventilated for three days. Broad-spectrum intravenous antibiotics (metronidazole and cefuroxime) were commenced for prophylaxis and he also received regular dexamethasone for the first 24 hours. Examination at extubation showed significantly reduced swelling and healing pharyngeal mucosal injury. The patient made an uneventful recovery and was discharged home five days later.

\section{Discussion}

Although pharyngeal injury resulting from a high-pressure water jet has not been reported, injuries to the rectum, vagina, limbs, nose, eyes and abdomen have been reported. The high-pressure of the water jet causes infiltration of water and air into the tissue planes, resulting in significant submucosal injury, as seen in this case and also described in water jet injuries to the rectum and vagina. The resultant soft tissue emphysema seen in this case appears as mixed radio-density on a radiograph, a pattern of injury similar to the subcutaneous emphysema seen in water jet injuries to the limbs. In addition to the primary injury caused by the kinetic energy of the water jet, ischaemia due to direct injury or thrombosis of blood vessels and the injection of bacteria leads to further inflammation. Thus, prophylactic antibiotics are an integral part of the management of water jet injuries.

\section{Conclusion}

This case shows the need for a high index of suspicion in assessing patients presenting with injuries from highpressure water jets. A patient presenting with a history of a direct hit in the mouth by a jet wash should be deemed to have impending airway obstruction until this is ruled out. Rapid evaluation and appropriate intervention including protection of the airway are required. 


\section{Positioning bone-anchored hearing aids in bilateral} microtia for optimal reconstruction

Y Bajaj, M E Wyatt, D Gault, C M Bailey, D M Albert From the Great Ormond Street Hospital for Children, London, UK

\section{Introduction}

Patients with bilateral microtia require both reconstruction of the auricle and rehabilitation of hearing. For implanting a bone-anchored hearing aid (BAHA) the thickest cortical bone is anteriorly near the microtic ear. For auricular reconstruction the BAHA needs to be implanted as posteriorly as possible. A compromise thus has to be reached to implant the BAHA as anteriorly as possible and not interfere with the auricular reconstruction. The aim of this study was to quantitatively analyse the optimal position for BAHAs in patients requiring reconstruction of the auricle on the same side.

\section{Method}

This was a prospective case study conducted in the Microtia Clinic at a tertiary level paediatric hospital. Ten patients with bilateral microtia and BAHA who consented for this study were included. For the purpose of this study, the position of the BAHA was measured from the bony external auditory meatus, angle of jaw and the angle of the eye. It was felt that the bony external auditory meatus was the most consistent landmark. Also for the purpose of this study special life sized photographs of these patients with grids for measurement were taken.

\section{Results}

Measurements were taken both on life sized photos and on the patients. The measurements on the photos and real time measurements were close but not the same, so for analysis the actual measurements on the patients were used. The distance of the BAHA abutment from the bony external meatus varied from $4.5 \mathrm{~cm}$ to $9.6 \mathrm{~cm}$, the average distance being $6.54 \mathrm{~cm}$. In most of the patients the distance from the BAHA to the external auditory meatus was between 6.5 and $7.0 \mathrm{~cm}$. In three of these 10 patients, the position of the BAHA was not correct for auricular reconstruction and had to/will need to be resited. In another two patients the BAHA was too close to the auricular remnant and not optimal for reconstruction. Altogether in five of these 10 patients (50 per cent) the positioning of the BAHA was not optimal for auricular reconstruction.

\section{Conclusions}

The authors would not recommend reliance upon photographs alone for making decisions about surgery, but they are certainly a useful adjunct. From this study it can be suggested that siting a BAHA 6.5 to $7.0 \mathrm{~cm}$ from the position of the external auditory meatus is probably the correct distance to facilitate optimal auricular reconstruction. Also, it is better to delay implanting a BAHA, and persevere with a bone conduction hearing aid, than to implant early in a suboptimal position.

\section{Surgical excision for subglottic haemangioma}

\author{
Y Bajaj, B E J Hartley, D M Albert, C M Bailey \\ From the Great Ormond Street Hospital for Children, \\ London, UK
}

\section{Introduction}

Subglottic haemangioma is an important cause of stridor in young children. It typically presents within the first few weeks or months of life. Subglottic haemangioma is a potentially life-threatening condition for which various treatment modalities are available. The treatment options for large subglottic haemangiomas include steroids (systemic or intralesional), laser ablation, interferon, open excision or tracheostomy. The objective of this study was to evaluate our results and share our experience of open excision of subglottic haemangiomas.

\section{Method}

This study included 17 patients who were treated at a paediatric tertiary referral centre. In all the cases the diagnosis was confirmed on microlaryngoscopy. The surgery was performed either as a single-stage or two-stage procedure. The single-stage procedure involved only intra-operative tracheostomy and post-operative intubation for seven days. In the two-stage procedure a tracheostomy was performed as the first step followed by elective surgical excision and then decannulation at a later date. Most of the patients undergoing surgical excision had a large subglottic haemangioma occluding $70-80$ per cent of the subglottis. Most of these children (82 per cent) had received steroids before surgery. The average age at the time of operation in this series was seven months (range two to 29 months). None of these patients had undergone any laser treatment before the operation.

\section{Results}

Most of these patients (14/17; 82 per cent) underwent open surgical excision as a single-stage procedure. In most of these patients $(12 / 17 ; 71$ per cent) an anterior cartilage graft was used for reconstruction. The average follow up in this study was 25 months. All the patients in this study had an adequate airway after the procedure. The results of open surgical excision in this study were very encouraging. Sixteen out of 17 (94 per cent) patients either avoided tracheostomy or were decannulated as a direct result of surgery. One of these 17 patients ( 6 per cent) required a temporary post-operative tracheostomy for 13 months as the subglottis cleared and this case can be classed as partial success. No patient in this series developed post-excision subglottic stenosis which is a frequent complication (20 per cent) of laser application.

\section{Conclusions}

Our experience is that open excision is a highly successful 'one stop' treatment for subglottic haemangiomas that avoids prolonged use of steroids and multiple repeat endoscopic procedures. Surgical excision of subglottic haemangioma offers a fast and satisfactory treatment for a condition which can be of concern to parents and the treating physicians when treatment has to be prolonged over a period of months or years.

\section{John Evans Lecture The face of paediatric otolaryngology}

\section{$\mathrm{R} \mathrm{T}$ Cotton}

From the Department of Pediatric Otolaryngology - Head \& Neck Surgery, Children's Hospital Medical Center, Cincinnati, Ohio, USA

This lecture presents an overview of the clinical and academic mission of the Pediatric Otolaryngology Service at Cincinnati Children's Hospital Medical Center.

The hospital is dedicated to serving the healthcare needs of infants, children and adolescents, and to provide research and teaching programmes, which ensure delivery of the highest quality paediatric care to our community, 
the nation and the world. How does paediatric otolaryngology support this vision?

Clinically, the Department provides primary services to the immediate drawing area of the hospital that approximates about one and a half million people. In addition, we manage programmes intended for our secondary area of catchment, which is approximately four million. Such programmes include the Airway and Voice Centre, Sensorineural Hearing Loss and Hearing Impaired Clinic, and the Velopharyngeal Insufficiency Clinic. We supply consultative support to the Vascular Malformation Team and the Craniofacial Anomaly Team. In addition, we have a tertiary care referral for diseases of the airway, which are managed by a multi-specialty interdisciplinary team of healthcare professionals centred in the Aerodigestive and Sleep Centre. This lecture will provide an outline of our current practices within these programmes.

Academically, the Department is vigorous in its basic science and translational research endeavours, and also in clinical outcome studies. Current studies of academic interest will be presented and discussed.

\section{Serum IgE and IgE functional antibodies to house dust mite and mixed grass pollens found in two aged-matched cohorts of children with and without otitis media with effusion}

C J Coulson, T Plant*, M T Drayson*, A B Drake-Lee

From the Department of ENT, Queen Elizabeth Hospital, Birmingham, and the *Department of Immunology, University of Birmingham, UK

\section{Introduction}

The aetiology of otitis media with effusion is debated and is thought to include respiratory allergy. This hypothesis remains unproven.

\section{Objective}

To determine whether allergy has a role in the pathogenesis of otitis media with effusion.

\section{Method}

Fifty children who had $>30 \mathrm{~dB}$ hearing loss and otoscopic appearance of otitis media with effusion for three months were admitted for ventilation tubes. Children with Down syndrome, cleft lip and palate, ciliary abnormalities, known immunodeficiencies and cardiac abnormalities were excluded. Fifty children admitted for minor eye surgery, who had otoscopically normal ears and no history of middle-ear problems for the previous twelve months were used as controls. All children in both groups were between three and 10 years. Bloods were taken under anaesthesia. Total $\mathrm{IgE}$ and $\mathrm{IgE}$ radioallergosorbent tests to house dust mite and mixed grass pollens were measured by the Pharmacea Unicap 100 system.

\section{Results}

There was no statistical difference between the control and study groups for the total IgE. Six children from both study and control groups had a raised radioallergosorbent test to either allergen. There was no difference in the levels between each group.

\section{Conclusion}

The study does not support the role of allergy in the pathogenesis of otitis media with effusion.

\section{Maturation sutures for paediatric tracheostomy}

M F Craig, Y Bajaj, B E J Hartley
From the Great Ormond Street Hospital for Children, London, UK

\section{Introduction}

When performing paediatric tracheostomy, a simple vertical tracheal incision is used without removal of a window of tracheal wall (as is done in adults). The addition of maturation sutures from skin to trachea forms a mature stoma, which allows the simple replacement of the tube if accidentally displaced, avoiding a potentially fatal outcome.

\section{Method}

We carried out a retrospective case notes study to assess the complication rate using maturation sutures. Data obtained included age, indication, diagnosis, grade of surgeon, skin and tracheal incision, tube type and size used. Complications were recorded according to whether they occurred before (early) or after (late) the time of first tube change, as well as any problems at the first tube change.

\section{Results}

Forty-four patients were found where maturation sutures had been used, over a four-year period. Thirty-eight notes were complete and therefore reviewed. Average patient age was just under two and a quarter years; 21 patients were under one year of age. Indications were: airway obstruction in 25 patients (including 13 with subglottic stenosis), five with multiple diagnoses, cardiopulmonary three, neurological conditions three, pre-operative two.

Thirteen patients (38 per cent) had early complications (within seven days). One patient had an accidental decannulation where the tube was quickly replaced with no further complications. The most common complication was peristomal ulceration or local infection (seven patients, 21 per cent). Other complications were: surgical emphysema (two patients), blocked tube episodes (two), change of tube to one of more appropriate size (one), bleeding (one). There were no problems at the time of first tube change.

Ten patients (30 per cent) had late complications (after seven days). One patient ( 2.5 per cent) had an accidental tube displacement, again replaced with no further harm. Eight patients ( 20 per cent $)$ had other complications; the most common of these were suprastomal granulation, suprastomal collapse, and minor local infection at the tracheostomy site.

No patients died from tracheostomy related causes. Five died from other causes. There were no additional complications observed which could be attributed to the technique.

\section{Conclusion}

This study supports the use of maturation sutures as a worthwhile technique to perform with paediatric tracheostomy.

\section{Does suctioning cause mucosal injury of the distal trachea} and carina in paediatric tracheostomy?

M Elloy, J Cooke, D M Albert

From the Great Ormond Street Hospital for Children, London, UK

\section{Introduction}

Suctioning is an essential part of routine paediatric tracheostomy care. At home, this is performed for many children by their parents or caregivers, who are comprehensively trained. Reported complications of suctioning 
through a tracheostomy tube include tracheal injury, hypoxia, infection and atelectasis.

This study aims to assess if suctioning, as part of routine tracheostomy care, causes significant mucosal injury to the distal trachea or carina which is detectable on endoscopic examination.

\section{Method}

Endoscopic image records are held prospectively on a theatre image database for all microlaryngobronchoscopies performed. A review of the ENT theatre lists, over a sixmonth period, at Great Ormond Street Hospital was performed. All patients documented to have a tracheostomy, who underwent microlaryngobronchoscopy over this time period were included in the study. Patients with a history of tracheoesophageal fistula were excluded. Data were retrospectively collated for each patient from the theatre database, tracheostomy database and theatre image database. The endoscopic images recorded at microlaryngobronchoscopy were reviewed and assessed for evidence of injury to the distal trachea or carina.

Results

Fifty-one patients with tracheostomy underwent microlaryngobronchoscopy in the study period. Forty-eight patients were included in the study. Three were excluded due to a history of tracheoesophageal fistula. Twenty-eight patients (58.3 per cent) had a normal appearance of the distal trachea and carina. One (2.1 per cent) demonstrated evidence of distal tracheal injury. In 14.6 per cent (seven), the distal trachea and carina could not be assessed due to disease and in 4.2 per cent (two) the carina could not be visualised because of a stent. In 20.8 per cent (10) of the patients, there were no endoscopic images of the distal trachea and carina. Therefore, of the 29 patients where the distal trachea and carina were visualised and assessed, 3.4 per cent $(1 / 29)$ had evidence of potential suction related trauma.

\section{Conclusion}

Suctioning of paediatric tracheostomies can be performed in the community by appropriately trained parents and caregivers with minimal risk of mucosal injury to the distal trachea and carina.

\section{UK and Ireland experience of bone-anchored hearing aids in individuals with Down syndrome}

P Hans, P Sheehan

From the Manchester Children's University Hospitals, Manchester, UK

\section{Introduction}

The Down syndrome population has a significantly higher incidence of hearing loss than the general population. It has been shown that even mild hearing loss can affect language acquisition and development in children without coexistent learning difficulties. For children with Down syndrome, therefore, it is important that any hearing loss is identified and corrected rapidly and effectively in order for them to achieve their full developmental potential.

The authors were aware that individuals with Down syndrome had benefited from the bone-anchored hearing aid (BAHA) system, after traditional interventions had failed. There was no literature, however, to indicate the number of individuals with Down syndrome that had been provided with BAHAs. It was decided therefore to survey the experiences of all BAHA centres in the UK and Ireland that had provided a BAHA service to individuals with Down syndrome.

\section{Method}

A postal survey of all registered United Kingdom and Ireland BAHA centres.

Results

There was a 98 per cent response rate to the survey. Eighty-one centres in the United Kingdom and Ireland undertake BAHA surgery. Eighteen centres were identified to have undertaken BAHA surgery on individuals with Down syndrome. A total of 43 individuals were implanted. Twenty-four cases were under the age of 16 . Soft tissue complications were encountered in 21 patients (49 per cent). Osseointegration failures were encountered in four cases ( 9 per cent). There was a high level of satisfaction with the BAHA system amongst patients, parents and carers.

\section{Conclusion}

BAHA is a valuable method of amplification in children with Down syndrome. It should be considered not as a primary method of amplification, but as a fall back method, after conventional hearing aids have been supplied and management with ventilating tubes has failed to resolve the otitis media with effusion. The survey shows a high patient and carer satisfaction with the system, despite short term early soft tissue complications.

\section{The history of myringotomy and ventilation tube insertion}

S E Henney, M Morgan

From the ENT Department, Sunderland Royal Hospital, Sunderland, UK

\section{Introduction}

Myringotomy and ventilation tube insertion is the operation most commonly performed under general anaesthesia in the Western world. From as early as 1649 there have been reports of therapeutic perforation of the eardrum. The knowledge of the development of this commonly performed operation is important for thorough understanding and a basis for future developments to constantly improve patient care.

\section{Method}

A computerised literature search was performed to identify all reports of myringotomy/tympanostomy and ventilation tube/grommet insertion from the Medline and Embase databases. Standard otorhinolaryngology textbooks were evaluated.

Result

The first report of piercing an eardrum, which improved patient hearing, was in 1649. Jaen Riolan the Younger accidentally injured the tympanic membrane when cleaning the external auditory canal with an ear spoon. Throughout the seventeenth and eighteenth centuries experiments by Valsalva et al. attempted to determine the function of the eardrum and hearing. At the end of the eighteenth century tympanic membrane perforation was indiscriminately being performed across Western Europe. It was Astlee Cooper in 1800 who first described an indication for myringotomy, obturation of the eustachian tube.

Following poor results with indiscriminate use of myringotomy, the operation lost favour. It was Herrmann Schwartze in Halle who successfully reintroduced the procedure. Spontaneous healing of the tympanic membrane 
was observed and various techniques were employed to maintain a permanent perforation. These included putting a foreign body into the aperture such as lead wires, whalebone and catgut.

In 1845 Martell Frank first described a grommet using gold foil. Other materials such as hard rubber and aluminium were used but again poor results led to the decline of the operation. Armstrong reintroduced vinyl grommets in 1954 for chronic secretory otitis media. This technique has been in use ever since with only minor refinements.

\section{Conclusion}

Myringotomy and ventilation tube insertion is a common and often successful treatment option with a long history. Considering 'historical' practice may be of benefit when developing new therapies.

\section{The presence of hyperacusis in children with primarily otological symptoms}

N Khan, J Hobson, B Lesniewicz, D Willatt

From the Royal Manchester Children's Hospital, Manchester, UK

\section{Introduction}

The term hyperacusis denotes increased sensitivity to sounds at a level that would not trouble a normal individual. In the same context, other terms such as phonophobia, noise sensitivity, hyperacusis dolorosa, and audio sensitivity have been used to highlight the same disorder. The aetiology is multifocal and can be caused by pathological problems in the central nervous system, the peripheral vestibule-cochlear apparatus or both. Head injury, endocrine problems and infections are among the causative factors implicated in children. The incidence of hyperacusis in children with otological problems is unknown. Its presence may be overshadowed by more pressing problems like otalgia, deafness and otorrhoea. In this prospective study, we aim to determine whether hyperacusis is present in children presenting to the ENT clinic with primarily otological symptoms.

\section{Method}

Seventy-one consecutive children who came to outpatients with ear complaints were included in this prospective study. The parent/guardian of the child was asked whether his/her child had abnormal discomfort to sound that did not annoy healthy individuals. In the patients with hyperacusis, the symptom was further explored with their parent/guardian by way of a questionnaire about their child's hypersensitivity to sound and its other various attributes. These children also had pure tone audiometry and/or tympanometry.

\section{Results}

Parents of 18 (25.31 per cent) of the patients described the presence of hyperacusis in their children. These 18 children were aged between two years eight months to eight years 10 months (mean age five years nine months). Of the children with hyperacusis, 17 ( 84 per cent) were sensitive to loud music while six (33 per cent) were sensitive to all loud sounds. Ten (55 per cent) regarded loudness as being painful to their ears. Sixteen ( 88.88 per cent) had some degree of hearing impairment ( $<30 \mathrm{~dB}$ on audiograms). Fourteen (77 per cent) regarded the hearing impairment as more annoying than hyperacusis. Tinnitus was found in two (11.11 per cent). Sixteen (88.88 per cent) had to modify their lifestyle to counter this problem. Since first noted, hyperacusis persisted in 14 (77 per cent) of the patients.
Of the children with hyperacusis who had tympanometry, 15 (82 per cent) had abnormal tympanograms. Of these 10 (66 per cent) had type B and five (33 per cent) had type $\mathrm{C}$ tympanograms.

\section{Conclusion}

Hyperacusis exists in about a quarter of patients with ear problems. This complaint should be enquired about as it is under reported by parents, guardians and children themselves. It may have some association with middle-ear dysfunction. Further research is needed to explain this problem in the paediatric age group.

\section{Immediate lamina papyracea reconstruction following endoscopic sinus surgery for surgically managed subperiosteal abscesses in children}

S Khwaja, V Malik, J D Carpentier

From the Royal Preston Hospital, Fulwood, Preston, UK

\section{Introduction}

The sinonasal area of a child's face is the keystone of facial architecture and any trauma to this area may result in facial dysplasia. Animal studies have already proven facial skeletal growth retardation following functional endoscopic sinus surgery.

The aim of this study was to investigate the benefit of lamina papyracea reconstruction in the case of endoscopic sinus surgery in children in order to reduce the risk of facial dysplasia.

\section{Method}

A prospective study over twelve months was carried out. Two children who presented with a subperiosteal abscess secondary to acute sinusitis and failed medical treatment were studied. Both of these patients had an endoscopic sinus procedure to drain the abscess and then proceeded to have an immediate reconstruction of the lamina papyracea.

\section{Results}

Both patients' post procedure computed tomography scan showed resolution of the abscess and no signs of dysplasia.

\section{Conclusion}

We recommend immediate lamina papyracea reconstruction following endoscopic sinus surgery for surgically managed subperiosteal abscess in children. The aim is to restore the facial skeletal anatomy and decrease the risk of long-term facial dysplasia.

\section{Hearing outcomes in children with primary ciliary dyskinesia - a longitudinal study}

A Majithia, J Fong, M Hariri, J Harcour

From the Department of Otolaryngology, Charing Cross Hospital, London, UK

\section{Introduction}

Primary ciliary dyskinesia is a congenital abnormality of ciliary structure or function. The otological manifestations of the disease include otitis media with effusion. To date the severity of hearing loss and natural progression of otitis media with effusion in this select group of patients has not been documented.

Method

In this retrospective observational study, we looked at the tympanograms and audiograms of all children with 
primary ciliary dyskinesia attending the Royal Brompton Hospital multidisciplinary clinic.

Results

Our results show an improvement in both hearing thresholds and tympanograms with age $(p<0.001)$. Most cases resolve by the age of 12 .

\section{Conclusion}

This supports the current practice of conservative management in these patients. The problems of persistent otorrhoea and residual tympanic membrane perforation are thereby avoided with the reassurance that hearing loss will spontaneously resolve with time.

\section{Food allergy and rhinitis - fact or fiction?}

\section{Malik, S Ghosh, T J Woolford}

From the Department of Otorhinolaryngology, Manchester Royal Infirmary, Manchester, UK

\section{Introduction}

Food allergy is often implicated as a cause of rhinitis in children especially by the parents. There is a lot of contradicting information available on the Internet.

\section{Method}

We have reviewed the available literature, which shows the incidence of food allergy in children to be $2-8$ per cent.

Results

The children usually grow out of food allergy with age. Food allergy usually presents with multi system involvement, most commonly cutaneous and gastrointestinal symptoms whereas rhinitis is less common. Isolated rhinitis due to food allergy is extremely rare. Diagnosing specific food allergens is difficult.

\section{Conclusion}

Treatment for rhinitis due to food allergy is usually not necessary as the incidence is not only quite low but also decreases with age.

\section{Vascular rings and slings causing tracheo-bronchial airway compression}

B McMonagle, G Morrison, S Blaney

From Guy's \& St Thomas' Hospitals, London, UK

\section{Introduction}

Airway obstruction may be the result of congenital cardiac and aortic arch anomalies. Extrinsic compression can be caused by a double aortic arch, a right arch with aberrant left subclavian artery, a left pulmonary artery sling, dilated pulmonary arteries, left atrial enlargement and cardiomegaly. In order to describe the characteristic clinical and endoscopic features and recommend management in infants with vascular compression of the airway, a retrospective review was undertaken.

\section{Method}

Over a five-year period, from June 1999 to June 2005, 28 infants were assessed by microlaryngoscopy and bronchoscopy with findings which suggested extrinsic vascular compression at the tracheo-bronchial level. In 23 of these the definitive diagnosis was a compressive vascular ring or pulmonary artery sling. Data were obtained from our paediatric ENT surgical and paediatric intensive care unit databases and clinical hospital records.

\section{Results}

The indication for microlaryngoscopy and bronchoscopy was stridor/recession, cyanotic episodes or because of failed extubation. Of 28 microlaryngoscopies and bronchoscopies which suggested a vascular compression, that diagnosis was confirmed after imaging in 23 , but no abnormal vasculature was found in five patients, (an 18 per cent false positive rate for endoscopic diagnosis). In four out of 23 (17 per cent) infants the diagnosis of vascular ring or sling was first suspected at diagnostic microlaryngoscopy and bronchoscopy despite a prior normal echocardiogram.

Other investigations included contrast swallow, chest computed tomography scan or chest magnetic resonance imaging (MRI) scan. Of the 23 infants, 13 had a double aortic arch, eight had a left pulmonary artery sling and one had severe left bronchomalacia from total anomalous pulmonary venous connection. Characteristically the double aortic arch is seen at microlaryngoscopy and bronchoscopy as a large pulsatile swelling posterior to the trachealis in the supracarinal trachea, occluding the airway. The malacia is associated with copious distal secretions. The features of a left pulmonary artery sling are more variable, also with posterior or left posterolateral pulsatile compression, and sometimes with complete tracheal rings and distal microtrachea. Definitive treatments are cardiothoracic surgical correction of the anomaly. Of the eight patients who underwent surgery for pulmonary artery sling, three had associated congenital long segment microtracheas. None required a tracheostomy. Of the 13 babies who underwent correction of a double aortic arch, three ( 23 per cent) required a tracheotomy for persistent supracarinal tracheomalacia, post-operatively. Two of these were later successfully decannulated of tracheostomy by 16 months of age. The infant who had total anomalous pulmonary venous connection despite cardiac surgery had severe life-threatening left bronchomalacia and was managed with endoscopic placement of a 'Palmaz' expandable metallic stent in the left main bronchus. The overall mortality in this series of infants was four out of 23 (17.4 per cent), three with left pulmonary artery sling and only one with a double aortic arch died.

\section{Conclusion}

Infants with stridor must be assessed at microlaryngoscopy and bronchoscopy. Echocardiography can miss the diagnosis of a major vessel anomaly in the chest. A double aortic arch and left pulmonary artery sling are the most common anomalies found, which can present with airway obstruction, the microlaryngoscopy and bronchoscopy features are described. Three-dimensional MRI scanning is recommended and even after cardiothoracic surgery the malacic trachea may require stenting with a tracheostomy before the condition resolves. Babies with left pulmonary artery slings, especially if associated with congenital microtrachea carry a higher mortality.

\section{Does hydrogen peroxide mouthwash prevent surgical intervention in paediatric secondary post-tonsillectomy bleed? A ten-year review}

I Pai, S Lo, S Brown, A Toma

From the Department of Otolaryngology, St George's Hospital, London, UK

\section{Introduction}

The reported incidence of secondary post-tonsillectomy haemorrhage in paediatric patients in the United Kingdom, defined as that occurring more than 24 hours after surgery, ranges from 1 per cent to 5.4 per cent. Most cases are managed conservatively with only between 
4.3 per cent and 8.0 per cent requiring surgical intervention. Many otolaryngologists in the UK prescribe hydrogen peroxide $\left(\mathrm{H}_{2} \mathrm{O}_{2}\right)$ mouthwashes for secondary posttonsillectomy haemorrhage. No studies have been reported in the medical literature to date.

\section{Method}

A 10-year retrospective chart review of patients aged 16 years or younger admitted to St George's Hospital between January 1994 and December 2003 with secondary post-tonsillectomy haemorrhage. Subjects were divided into two groups depending on whether or not they had received hydrogen peroxide mouthwash. The main outcome measures were the duration of hospital admission following secondary haemorrhage, surgical intervention for arrest of haemorrhage and readmission with further haemorrhage. Statistical analysis was carried out using the $t$-test and Wilcoxon rank sum test.

\section{Results}

Fifty-nine patients received $\mathrm{H}_{2} \mathrm{O}_{2}$ and 97 did not. All patients received broad-spectrum intravenous antibiotics. The mean age was just 7.01 years (range 1.25 to 13.14 years). The average length of hospital stay was 1.6 days (control) and 1.7 days $\left(\mathrm{H}_{2} \mathrm{O}_{2}\right.$ group) respectively. Eight and a half per cent $(n=5 / 59)$ of the $\mathrm{H}_{2} \mathrm{O}_{2}$ group required surgery (all within 24 hours of admission), compared to 10.3 per cent $(n=10 / 97)$ of the control group (nine within 24 hours, one within 48 hours). The average number of doses of $\mathrm{H}_{2} \mathrm{O}_{2}$ prior to surgery was 1.8. Three point four per cent $(n=2 / 59)$ of the $\mathrm{H}_{2} \mathrm{O}_{2}$ group and 3.1 per cent $(n=3 / 97)$ of the control group were readmitted with further haemorrhage following their first episode of secondary haemorrhage. There was no statistically significant difference between the two groups in the length of hospital stay ( $t$-test $p=0.49$ ), the rate of surgical intervention (rank sum test $p=0.85$ ), or the rate of readmission for recurrent haemorrhage (rank sum test $p=0.92$ ).

\section{Conclusion}

$\mathrm{H}_{2} \mathrm{O}_{2}$ mouthwash does not seem to influence the outcome of secondary post-tonsillectomy haemorrhage in paediatric patients. A prospective randomised controlled trial is recommended to further elucidate our findings.

\section{Softband ${ }^{\circledR}$ - an alternative treatment for glue ear}

Y Ramakrishnan, T Davison, I Johnson

From the Freeman Hospital, Newcastle Upon Tyne, UK

\section{Introduction}

Bone-anchored hearing aids (BAHAs) have been used since 1977 for conductive and mixed hearing loss, and recently in 2002 for single-sided sensorineural deafness. A variant of this called Softband ${ }^{\circledR}$ was introduced in 2002 for babies less than three years, giving them the opportunity to experience the benefits of the BAHA system until they are old enough to have surgery. For years, Softband ${ }^{\circledR}$ has only been used for fixed conductive hearing loss conditions. This study is the first of its kind nationwide to investigate the benefit of Softband ${ }^{\circledR}$ in schoolchildren with fluctuating conductive hearing loss due to otitis media with effusion.

\section{Method}

This is a prospective, non randomised study, which took place at the Freeman Hospital, Newcastle, which is the largest BAHA centre nationwide, between 2004 and 2005. It is a self-selected group comprising 11 patients, aged between six and 15 years (mean age 9.3 years). The male:female ratio was four:seven. They all suffered from otitis media with effusion with conductive loss of $30 \mathrm{~dB}$ on average and had normal sensorineural hearing. They opted for Softband ${ }^{\circledR}$ having either failed treatment with ventilation tubes or declining surgery.

Two questionnaires were sent to be completed by the parents - the Modified Listening Situations questionnaire and Freeman questionnaire (see appendix). The modified Listening Situations questionnaire is validated and is already used by teachers of the deaf while the Freeman questionnaire is a non-validated open test. The former assigns scores of zero to four for each question (zero being always easy and four being most difficult) giving a minimum score of zero and a maximum score of 44 . The Freeman questionnaire is not scored but gives an impression of the benefit derived from Softband ${ }^{\circledR}$ at home, school and outdoors.

\section{Results}

Of the 11 questionnaires sent out, 10 were returned giving a response rate of 90.9 per cent. The average modified Listening Situations questionnaire score was 13.3 $(3,4,6,9,11,15,17,19,19,30)$.

The Freeman questionnaire showed that $9 / 10$ children used the Softband ${ }^{\circledR}$ regularly whilst the remaining one used the Softband ${ }^{\circledR}$ as a back-up during episodes of otitis media with effusion. All of them used the Softband ${ }^{\circledR}$ at school, one outdoors and two at home. Six of the 10 used it for up to six hours daily whilst three of the 10 wore it for more than six hours.

Six of the 10 teachers noticed an improvement whilst three of the 10 were more responsive at home. Peer interaction was improved in four out of 10 cases. In two of the 10 cases, speech improved. There were no side effects related to the Softband ${ }^{\circledR}$.

\section{Conclusion}

This trial is the first of its kind to show that Softband ${ }^{\circledR}$ can be used for treatment of glue ear. It benefits from being easy to self-administer, is non-invasive and has no side effects. Future national trials will be necessary to assess the full benefit of Softband ${ }^{\circledR}$ in otitis media with effusion.

\section{An effective alternative to traditional skin closure in paediatric ear surgery}

V Nandapalan, S R Ramasamy, T H J Lesser, O A Ochefu From the Department of Otolaryngology, Walton Hospital, Liverpool, UK

\section{Introduction}

The aim of the present study was to evaluate the applicability, effectiveness and adverse effects of the tissue glue as an alternative to traditional skin closure in children undergoing ear surgery.

\section{Method}

Fifteen children aged between eight and 12 who were undergoing major ear surgery were recruited. After adequate subcutaneous closure, the histoacryl glue was used to approximate the skin instead of sutures. They were followed up at the end of the first and fourth week postoperatively to assess the state of the wound, complications, cosmetic appearance and patient satisfaction.

Results

At the end of the first week no wound infection or dehiscence was noted. In three of the children who had end aural incision the wick was adherent to the wound. At the end of four weeks all incisions were healed well with excellent cosmetic appearance. All parents were delighted 
with both the trouble-free post-operative period and the cosmetic result.

\section{Conclusion}

Tissue adhesives are an effective alternative to traditional skin closure in paediatric otology. The parents were delighted as this procedure was both cosmetic and obviated the need for suture removal.

\section{Role of otoacoustic emissions in children with middle-ear effusion and grommets}

Y Saleem, S Ramachandran, L Rammamurthy, N J Kay From the Department of Otolaryngology, Stepping Hill Hospital, Stockport, UK

\section{Introduction}

For screening of middle-ear effusion and follow up of grommets, pure tone audiometry and tympanometry are widely accepted. In young and difficult to test children this could be labour intensive and time consuming. Otoacoustic emissions using transient evoked otoacoustic emissions are relatively quick and simple to perform. Many of the initial attempts to examine the practical utility of emitted responses revealed that the non-invasive properties of the test were promising in the testing of infants and young children. It is clear that young patient populations would benefit from emission testing and the feasibility of such an application in a group of subjects with middle-ear effusion has not yet been adequately studied. The aim of our study is to determine the role of otoacoustic emissions in children with middle-ear effusion and grommets.

\section{Method}

In this prospective study of patients undergoing grommet insertions for middle-ear effusion, 90 ears were studied. The age group of the patients was between two and 15 years. Patients diagnosed with middle-ear effusion and listed for grommet insertion were included in the study. Pre-operatively all patients had a pure tone audiometry, tympanometry and otoacoustic emissions done and recorded. Patients were seen three to six months after the grommet insertion and again pure tone audiometry, tympanometry and otoacoustic emissions were recorded. All ears were examined prior to the tests and only ears with grommets in situ and patent grommets were included in the study.

\section{Results}

Out of the total 90 ears studied, pre-operatively 63 ears showed evidence of conductive loss on pure tone audiometry and the remaining 27 ears had normal hearing. Of the 27 ears with normal hearing, 21 had a type A tympanogram, three had a flat type B tympanogram and three had a type $\mathrm{C}$ tympanogram. Of the 63 ears, which had a conductive loss, 27 had a type $\mathrm{A}$ and 17 had a type $\mathrm{C}$ tympanogram and the remaining 21 ears had a type B tympanogram. Fifty-nine of the ears pre-operatively had an absent/ reduced otoacoustic emission and the remaining 31 ears had otoacoustic emissions present. All the 59 patients who had an absent/reduced otoacoustic emission had a conductive hearing loss on pure tone audiometry and 27 of the 31 ears that had an otoacoustic emission response had a normal pure tone audiometry. There was a strong correlation between pure tone audiometry and otoacoustic emissions pre- and post-operatively.

\section{Conclusion}

We found that both pure tone audiometry and otoacoustic emissions were closely correlated both in patients with middle-ear effusion and in patients with grommets.
We conclude that otoacoustic emissions can be used as an alternative to pure tone audiometry and tympanometry in patients with middle-ear effusion and grommets.

\section{Xanthoma disseminatum revisited}

R K Sharma, C H Raine

From the ENT Department. Bradford Royal Infirmary Teaching Hospitals, Bradford, UK

\section{Introduction}

Xanthoma disseminatum, also known as Montgomery's syndrome is a benign non-inherited, normolipaemic, mucocutaneous, non-Langerhans cell histiocytosis. It is characterised by papular cutaneous eruption with a predilection for flexure creases. Mucosal involvement is present in almost half the cases. There is frequent association of vasopressin-sensitive diabetes insipidus due to xanthoma deposits in the hypothalamus-pituitary axis.

The case 'AM' was presented to the BAPO in 1997. The patient had palatal scarring, poor tongue mobility, marked supraglottic stenosis and paralysed vocal folds at presentation in 1995 . He required an urgent tracheotomy a year later.

The purpose of this presentation is to highlight the spontaneous resolution of various aspects of this condition and a request for advice in further management.

\section{Case report}

AM was a 12-year-old Asian child when he was referred with hoarseness, mild stridor and dyspnoea on exertion in 1995. He had been diagnosed with xanthoma disseminatum, with multiple brown papular eruptions in the face, conjunctiva and the corneal surface of both eyes, compromising his eye-sight. There was no family history of note. On examination, there were xanthomas in his mouth, pharynx, larynx and hypopharynx. He had bilateral fixed vocal folds and poor vision (of 6/36). In 1996 he required an urgent tracheotomy, which remains in situ. Excision of the corneal granuloma with corneal grafting was done in the year 2000 for the right eye. The patient's skin lesions and vision (6/ 6) started to improve spontaneously in 2001 . The possibility of reversal of tracheotomy was contemplated. In 2002, airway endoscopy revealed a marked supraglottic stenosis (admitting $4 \mathrm{~mm}$ telescope), aryepiglottic web and limited abduction of the vocal folds. The patient underwent four procedures to widen the supraglottis using a $\mathrm{CO}_{2}$ laser, care being taken to avoid excising the arytenoids, but it tends to re-stenose. At present, a decade after presentation, his vocal folds are mobile. He still requires a tracheotomy because of supraglottic restrictions. His facial features have improved and the skin has smoothened. Despite the scarring of the soft palate his swallowing is much better.

\section{Conclusion}

Xanthoma disseminatum is potentially a life threatening, disfiguring and multi system disease but it is perhaps best treated with minimal intervention as spontaneous resolution is likely to occur even after many years. Long-term follow up is essential.

\section{Post-operative high dependency unit monitoring for obstructive sleep apnoea syndrome - is it necessary?}

R Sharma, A Dippanaar, N Bateman, P D Bull

From the ENT Department, Sheffield Children's Hospital, Sheffield, UK

\section{Introduction}

Obstructive sleep apnoea syndrome in children is most often due to tonsil and/or adenoid hypertrophy. Children 
with craniofacial anomalies and complex heart and lung conditions may have other contributory factors. The first line treatment for obstructive sleep apnoea syndrome in the majority of children remains adenotonsillectomy. There is a generally held opinion that children with obstructive sleep apnoea syndrome undergoing surgery require close observations post-operatively in high dependency or intensive care units. This results in many such patients being transferred to hospitals having intensive care beds and increased pressure on these facilities. In this study we retrospectively assessed the need for high dependency monitoring in a cohort of our patients who had undergone adenoid/tonsil surgery for obstructive sleep apnoea syndrome.

\section{Method}

The case notes of 22 patients admitted to the high dependency unit post-operatively for observations over a 12-month period from January to December 2004 were reviewed retrospectively. All had undergone adenotonsillectomy and had documented obstructive sleep apnoea syndrome. The data collected included pre-operative booking of the high dependency unit bed, presence of other cardiorespiratory pathology, abnormal signs or monitoring while on the unit, airway intervention and duration of stay on the unit.

\section{Results}

High dependency unit monitoring was utilised for 20 patients while two patients were admitted to the intensive care unit. Other cardiorespiratory or central pathology was present in five patients. Surgery was initially postponed due to lack of bed on the day of surgery for seven patients. Three patients required airway support: one by mask and two by endotracheal intubation and ventilation. All of these patients had coexisting morbidity in the form of craniofacial abnormalities and/or cardiopulmonary pathology. Two patients were discharged to the ward on the same day, sixteen patients the next day and one after four days. There was one death related to cardiorespiratory problems.

\section{Conclusion}

We have observed that the majority of our patients required no intervention during their stay in the high dependency unit post-operatively. Those that required ventilatory support had easily identifiable comorbidity. Based on these findings we would suggest that it would be appropriate to revisit the policy of admitting children with suspected obstructive sleep apnoea syndrome to a high dependency unit after adenotonsillectomy.

\section{British Association of Paediatric Otolaryngology members' experience with recurrent respiratory papillomatosis}

R A Tasca, R W Clarke, M S McCormick

From the ENT Department, Alder Hey Children's Hospital, Liverpool, UK

\section{Introduction}

Recurrent respiratory papillomatosis is the most common neoplasm in the larynx and the second most common cause of hoarseness in children. Though benign in histology, epithelial proliferations may result in progressive hoarseness, stridor, airway obstruction and respiratory distress rendering recurrent respiratory papillomatosis a serious and potentially life threatening disease. The larynx is the most commonly affected site with distal tracheobronchial and pulmonary involvement rare. Current treatment aims to maintain a patent airway and acceptable voice, while preventing complications such as laryngeal scarring or stenosis. The objective of this survey is to evaluate the current surgical and medical management of recurrent respiratory papillomatosis in the UK, by surveying the members of the British Association of Paediatric Otolaryngology (BAPO).

\section{Method}

A postal survey regarding the experience with patients with recurrent respiratory papillomatosis was sent to 104 BAPO consultant members between December 2003 and March 2004. A further e-mail based reminder was administered during the month of April 2004. Membership affiliation was obtained from the BAPO register and cross-checked with the ENT UK members list. The questionnaire used was the same one as used by Prof Derkay for his American Society of Pediatric Otolaryngology survey.

\section{Results}

Data were analysed from 39 respondents representing 26 departments (37.5 per cent response rate) for a total of 93 patients. A total of 11 patients (11.8 per cent) received adjuvant medical therapies with cidofovir accounting for eight patients. Distal spread of recurrent respiratory papillomatosis has occurred in 24 ( 25.8 per cent) patients. There were six reported deaths due to progressive recurrent respiratory papillomatosis. Various lasers $\left(\mathrm{CO}_{2}, \mathrm{KTP}\right.$, and Pulsed Dye) are the preferred method of surgical removal of recurrent respiratory papillomatosis in children. Spontaneous ventilation ( 62.5 per cent) is the preferred method of anaesthesia. Half of the consultants do not routinely send for human papilloma virus subtyping and 75 per cent send lesions for histological examination if there is a change in growth pattern. Two thirds of respondents do not routinely treat their patients with antireflux medication.

\section{Conclusions}

Firstly, there is a need for the establishment of a centralised national database to which all treating consultants can report their cases. This will eliminate some of the biases in this survey. Secondly, the time has come for a national multicentre randomised controlled trial on the use of adjuvant cidofovir for the treatment of both severe and less severe recurrent respiratory papillomatosis disease. Both of the above can be organised under the umbrella of BAPO. Thirdly, treatment trends are largely similar on both sides of the Atlantic. Recurrent respiratory papillomatosis is a benign disease with significant morbidity and potential mortality. Treatment continues to be difficult and involves both surgical and medical modalities. Both modalities are continuously evolving in search of a 'cure' with decreased side effects. Only further research and international cooperation can potentially achieve these aims.

\section{Branchial sinus of the piriform fossa: reappraisal of third and fourth branchial anomalies}

\section{K T Tzifa, A James, C Patterson, V Forte}

From the Hospital for Sick Children, Toronto, Canada

\section{Introduction}

The hypothetical courses of third and fourth branchial anomalies are widely described in the literature. They arise in the pyriform fossa and are then expected to pass superiorly over the hypoglossal nerve (third arch) or inferiorly under the aortic arch (left fourth arch) or right subclavian artery (right fourth arch). These hypothetical courses are not consistent with anomalies treated at our institution. 


\section{Method}

We retrospectively reviewed charts of children with third or fourth branchial anomalies using hospital databases to identify cases treated at the Hospital for Sick Children between 1992 and 2005. Imaging, operation notes and histopathology were reviewed to confirm accuracy of the diagnosis.

\section{Results}

Eighteen cases of branchial lesions arising from the pyriform fossa were identified of which 17 were left sided. Typical presentation was with a recurrently infected neck abscess closely associated with the ipsilateral thyroid gland. None presented primarily as a fistula, though secondary fistula formation occurred following incision and drainage. Contrast enhanced computed tomography scans showed an inflammatory mass occasionally containing gas and associated with disruption of the ipsilateral thyroid lobe. At surgery, the tract was found to pass directly from an opening in the pyriform fossa through or close to the thyroid lobe and into the lower neck. None passed over the hypoglossal nerve or into the mediastinum. Excision of the lesion with ipsilateral hemithyroidectomy provided definitive treatment in all cases.

\section{Conclusion}

In our experience, branchial anomalies arising from the pyriform fossa present as an infected sinus and not as a congenital fistula. They do not follow the widely reported, but hypothetical, course of third and fourth arch anomalies proposed in the literature. We consider that these pyriform sinus anomalies are formed by descent of third, or possibly fourth, pouch derivatives through the branchial arches. Unlike the parathyroid derivatives of these pouches which are solid, the thymus descends from the third pouch leaving behind it the thymopharyngeal duct which is patent. In normal development, the lumen becomes obliterated after midline fusion of the thymus. It is plausible that branchial sinuses from the pyriform fossa result from failure of closure of the thymopharyngeal duct. Excision of the lesion up to the pyriform fossa with ipsilateral hemithyroidectomy is an effective treatment.

\section{Endoscopic treatment of congenital H-type and recurrent tracheosophageal fistula with electrocautery and histoacryl glue}

K T Tzifa, L Maxwell, P Chait, A James, V Forte

From the Department of Paediatric Otorhinolaryngology, The Hospital for Sick Children, Toronto, Canada

\section{Introduction}

Congenital H-type tracheoesophageal fistulae and recurrent fistulae after primary repair of oesophageal atresia represent a difficult problem in diagnosis and management. The treatment traditionally involved an open technique via a cervical or thoracic external route, approaches with high morbidity and mortality rates of up to 50 per cent. Endoscopes have been used for the treatment of H-type tracheoesophageal fistulae and recurrent tracheoesophageal fistulae with lower post-operative complication rates. A variety of endoscopic methods have been described with diathermy or laser or adhesive materials. We present the experience at Toronto's Hospital for Sick Children since 1995, using diathermy and histoacryl tissue adhesive.

\section{Method}

Since 1995, 192 patients have been managed in this institution with tracheoesophageal fistulae of which ten patients have been treated endoscopically. Two fistulae were of
H-type, one traumatic and the rest (seven) were recurrent tracheoesophageal fistulae following surgery for oesophageal atresia and surgical division of tracheoesophageal fistulae. The procedure was undertaken under general anaesthetic in the diagnostic imaging suite under radiographic control. Flexible ball electrocautery and injection of histoacryl glue were used either on their own or in combination depending on the size of the fistula.

\section{Results}

All of the reported patients had a satisfactory outcome and closure of the fistula was achieved. In some cases (four) the procedure had to be repeated once more. Histoacryl glue overspill had to be removed and this was easily done endoscopically. No major respiratory or other complications were encountered. Follow up has been between three months to 10 years.

\section{Conclusion}

We conclude, endoscopic diathermy of tracheoesophageal fisulae with or without histoacryl glue has been a safe and successful technique of managing $\mathrm{H}$-type and recurrent tracheoesophageal fistulae with a maximum of up to two repeat procedures. Electrocautery has been reported before, sometimes with a bad outcome. In this paper we discuss the indications and the surgical steps of the procedure. We highlight that diathermy should be carefully controlled and applied preferably in the small non-patulous fistulae.

\section{Tongue-tie division in infants with breastfeeding difficulties}

H Wallace, S E Clarke

From the Pinderfields Hospital, Wakefield, UK

\section{Introduction}

Tongue-tie is a congenital oral abnormality characterised by an abnormally short lingual frenulum. Historically, tongue-ties were thought to affect breastfeeding and were commonly divided by the midwife at delivery. As formula milk gained popularity in the twentieth century, the technique fell out of favour as most infants with tonguetie can bottle feed successfully. With increased popularity of breastfeeding in the last decade there has been renewed interest in tongue-tie and its effect on breastfeeding. We present a case series of infants who underwent tongue-tie division for feeding difficulties.

\section{Method}

Eleven infants with breastfeeding difficulties associated with tongue-tie were identified by lactation consultants and seen in the next out-patient clinic by the second author for assessment and tongue-tie division using a standard technique. No anaesthetic or analgesia was used and there was little or no bleeding or infant distress. Follow-up on the effect of the procedure on feeding and any complications encountered was made routinely.

\section{Results}

The age at tongue-tie division ranged from two to 31 days (median 10 days). Ten of the 11 infants were followed up. The age at follow up was four to 20 months (median 10 months). Prior to division, all mothers had attempted breastfeeding and were keen to continue. Nine of the 10 had experienced difficulties due to poor latch $(8 / 10)$, sore nipples (6/10) and continual feeding cycle (5/10). Only three of the 10 mothers were breastfeeding exclusively; five were supplementing with bottle feeding and two were cup feeding. Following tongue-tie division, an 
improvement in breastfeeding was noticed immediately by four of the 10 mothers. Improvement took one to 14 days for three mothers. Three mothers did not notice any improvement. Six of the 10 mothers successfully breastfed for at least four months. One mother stopped breastfeeding after two months. Three mothers breastfed for less than one month. There were no reported complications of the procedure.

\section{Conclusion}

Lactation consultants are becoming more aware of tonguetie as a treatable cause of breastfeeding difficulty. This case series demonstrates the benefits of tongue-tie division in symptomatic infants with seven of the 10 mothers reporting an improvement in breastfeeding and six of the 10 mothers breastfeeding successfully for over four months. This is more than the national average for England and Wales of 29 per cent. Ideally, in the future, lactation consultants will be suitably trained and qualified to perform the simple procedure, thus reducing delay and hopefully increasing the number of infants successfully breastfeeding with all the advantages this brings.

\section{Ultrasound guidance of an obscured bone-anchored hearing aid fixture}

G Watson, H Hack, P Sheehan

From the Department of Otolaryngology, Royal Manchester Children's Hospital, Manchester, UK

\section{Introduction}

We present the innovative use of intra-operative real time ultrasonography to detect the position of a bone-anchored hearing aid (BAHA) fixture obscured by the overgrowth of scar tissue. This reduced theatre time considerably and avoided the use of ionizing radiation and the necessity to re-elevate the skin flap to reattach the BAHA abutment.

\section{Method}

A hand-held ultrasound probe (iLook $25^{\mathrm{TM}}$ Sonosite ${ }^{\circledR}$ ) was used to locate a BAHA fixture obscured by excessive scar tissue. This showed accurately as a large echogenic shadow within the skull and avoided unnecessary surgery and exposure to ionizing radiation.

\section{Conclusion}

Ultrasound has long been used for diagnostic procedures and to locate foreign bodies in obstetrics and gynaecology and general surgery. It has very rarely been used to locate foreign bodies in the head and neck region. The usage of ultrasound to locate an obscured BAHA fixture is a novel approach to this problem and should be considered in the future as it avoids radiation exposure, unnecessary surgery and is easy to use.

\section{Laser arytenoidectomy in children}

G Worley, Y Bajaj, L Cavalli, B Hartley

From the Great Ormond Street Hospital for Children, London, UK

\section{Introduction}

Vocal fold paralysis in children is considered to be the second most common congenital anomaly of the paediatric airway. Bilateral vocal fold immobility in children is a challenging problem because a balance between good airway and voice quality has to be achieved. Surgery to improve the airway is often postponed or avoided because of fear of losing the voice. The surgical management should aim to improve the patient's airway and at the same time avoid a breathy or weak voice. In this study our results of laser arytenoidectomy in children are described.

\section{Method}

This was a retrospective case notes review of six consecutive laser arytenoidectomies performed under the care of the senior author $(\mathrm{BH})$ at a tertiary level paediatric ENT department. All patients had bilateral vocal fold immobility. Four of these patients had a tracheostomy in situ at the time of the procedure. The surgery was performed by suspension microlaryngoscopy under general anaesthesia. The vocal process of one arytenoid and a small part of the anterior body of the arytenoid were removed using the laser. Care was taken to avoid injury to the interarytenoid mucosa. Care was also taken to leave the anterior two-thirds of the vocal fold undisturbed. The outcome measures were post-operative airway and voice quality.

\section{Results}

The patients in this case series were in the age range of nine to 16 years old at the time of arytenoidectomy. The average follow up for these patients was 18 months. All children in the series had an adequate post-operative airway. Four of these patients had tracheostomies pre-operatively and achieved decannulation. All six patients rated their postoperative voice as better than pre-operatively. This is principally due to increased loudness associated with increased airflow through the larynx, particularly after tracheostomy decannulation. None of the patients in this series needed a revision operation and there were no complications during or after the procedures.

\section{Conclusion}

Laser arytenoidectomy in children with bilateral vocal fold immobility, can produce good outcomes for both airway and voice. It is recommended that special care should be taken not to disturb the anterior two-thirds of the vocal fold during the surgery in order to achieve a good postoperative voice outcome. 